\title{
A CONSTITUIÇÃO DA CIÊNCIA DA CONSERVAÇÃO E A PROJEÇÃO DA CIÊNCIA DO PATRIMÔNIO
}

\author{
Yacy-Ara Froner \\ Professora UFMG, Coordenadora do LAPA-MHNJB-UFMG \\ froner@ufmg.br
}

\begin{abstract}
Resumo: A integração gradual da ciência no âmbito museológico e o fortalecimento da colaboração entre conservadores-restauradores, cientistas da conservação e curadores podem fornecer as pistas necessárias para compreender a história da Ciência da Conservação e a projeção atual da Ciência do Patrimônio. Este artigo tem como objetivo discutir a Primeira Conferência Internacional para o Estudo de Métodos Científicos para o Exame e Conservação de Obras de Arte, que ocorreu em Roma, em 1930. Realizado sob os auspícios do Escritório Internacional de Museus (1926-194), do Instituto Internacional de Cooperação Intelectual (1924-1946) e por meio das ações dos comitês nacionais, este encontro pode ser considerado um marco no estabelecimento da área da Ciência da Conservação, tanto no que se refere à visibilidade dos primeiros laboratórios, quanto no que concerne as discussões de formação do Conservador-restaurador.
\end{abstract}

Palavras Chave: ciência do patrimônio, ciência da conservação, conservação-restauro, epistemologia, formação

Abstract: SCIENCE CONSTITUTION OF CONSERVATION AND THE PROJECTION OF HERITAGE SCIENCE. The gradual integration of science into the museum and the strengthening of the collaboration between the conservators-restorers, the conservation scientists, and the curators describe the early history of the Conservation Science and the current launch of the Heritage Science. This paper aims to discuss the First International Conference for the Study of Scientific Methods for the Examination and Preservation of Works of Art, which took place in Rome 1930. It was held under the auspices of the International Museums Office (1926-1946), of the International Institute of Intellectual Co-operation (1924-1946), and of the Nationals Committees. Also, it must be realised as the landmark in the discussion of the Conservation Science of the Cultural Heritage because of the visibility of the first laboratories, besides of the establishment of the debates about to the conservator-restorer training.

Keywords: heritage science, conservation science, conservation-restoration, epistemology, training

\section{INTRODUÇÃO}

A história da Ciência do Patrimônio e a formação epistemológica das disciplinas que compõem sua estrutura - Ciência da Conservação, Conservação Preventiva, História da Arte Técnica, Arqueometria, Métodos e Técnicas de Conservação e Restauro de Bens Móveis, Métodos e Técnicas de Conservação e Restauro de Bens Imóveis, entre outras - têm sido objeto de muitas investigações e pesquisas (BALLESTREN, 1978; JOKILEHTO, 1986; PRICE, 1996), bem como revisões recentes (SEASE, 1996; FRONER, 2001; WHITMORE, 2011; LEVEAU, 2012; CATALANO, 2013). Por sua vez, a definição da área de 'Ciência da Conservação' tem sido construída por inúmeras publicações, desde sua primeira utilização em 1930. Em 2013, um grupo de especialistas reunidos em Roma debateram amplamente o conceito e o resultado dessas reflexões foram publicadas recentemente sob o título "Conservation Science" (ICCROM, 2015).

Questões relacionadas ao reconhecimento científico, acadêmico e profissional da área de Ciência da Conservação e do campo formação do conservador-restaurador de Bens Culturais, assim como as implicações políticas e sociais no que tange à preservação do Patrimônio Cultural em distintos contextos, podem ser observadas em uma conjuntura internacional de longa duração.

Esta comunicação propõe o deslocamento temporal do paradigma que reforça o nascimento da

doi: $10.18285 /$ geonomos.v24i2.838
Ciência da Conservação na segunda metade do século $X X$ para a primeira metade do século $X X$, considerando as primeiras conferências, a fundação dos primeiros laboratórios (Tabela 1), a discussão sobre a formação do conservador-restaurador (EIGENBERGER et ali, 1932; LEVEAU, 2011; FIGUEIRA, 2015) e o aparecimento do conceito de Ciência da Conservação pela primeira vez na revista "Mouseion", uma publicação organizada pelo Escritório Internacional de Museus e pelo Instituto Internacional de Cooperação Intelectual da Liga das Nações entre 1927 e 1946. Além disso, entende que as atividades do cientista da conservação e do conservador-restaurador de bens culturais estão inter-relacionadas, e que o mapeamento da origem desta relação no início do século XX pode contribuir ao debate.

\subsection{Formação e cooperação intelectual no} período entre-guerras (1919-1939

Nos séculos XVIII e XIX, as primeiras faculdades visaram à formação de uma elite burguesa em distintos países. O bacharel, geralmente circunscrito a um grupo restrito, assumiu um papel fundamental no contexto social. Investida de um capital simbólico do conhecimento, a formação - postulada por uma base positivista e utilitária - determinava a hierarquia desses indivíduos em sistemas específicos da Política, da Cultura e da Ciência. Herdeira das profundas alterações no projeto educacional europeu - como a criação das Ècole Polytechnique (1794), Ècole Normale (1794) e do Conservatoire dês Arts et Métiers (1794) a partir da Revolução 
Francesa e das reformas napoleônicas - a formação nos campos da Arquitetura, da Arqueologia, da Conservação, da Museologia, da História da Arte e da Arte compartilhou o território de gênese das Belas Artes (LAURENT, 1999; LEVEAU, 2008). Da mesma forma, distintas disciplinas das Ciências Naturais encontraram seus caminhos preliminares nos estudos politécnicos e nas ciências dos materiais, principalmente no que compete a função utilitária do saber na Revolução Industrial e no sistema capitalista (CLARK, 1993). A conformação da pesquisa em torno do Patrimônio Cultural gerenciou nesse sistema, portanto, sua vinculação a campo interdisciplinar de conhecimento.

Tabela 1. Laboratórios de Museus, Institutos Politécnicos e Universidades vinculados aos conferencistas - Fonte: Biblioteca dell'Accademia dei Lincei e Corsiniana, Fondo Francesco Pellati, Caixa 6, 171, (1930).

\begin{tabular}{|c|c|c|}
\hline Conferencista & Conferência 1930 & Laboratório \\
\hline $\begin{array}{l}\text { 1. BAUD-BOVY, Daniel } \\
(1870-1958)\end{array}$ & $\begin{array}{l}\text { "De l'utilité de la radiographie à propos d'un tableau du peintre genevois } \\
\text { de la Rive» } \\
14 \text { Octobre - Martin Section A. Peinture }\end{array}$ & $\begin{array}{l}\text { Laboratoriedu conservation du Musée Rath (Suisse) } \\
\text { (1905) }\end{array}$ \\
\hline $\begin{array}{l}\text { 2. BRITTNER, Carl (1883- } \\
\text { 1958); } \\
\text { RUHEMANN, Helmuth } \\
\text { (1891-1973) }\end{array}$ & $\begin{array}{l}\text { «Procédée de nettoyage des sculptures en pierre» } \\
15 \text { Octobre - Mardi Section B. Sculpture }\end{array}$ & $\begin{array}{l}\text { Laboratoire de Chimie aux Musées d'Etat à Berlin. } \\
(1888-1943)\end{array}$ \\
\hline $\begin{array}{l}\text { 3. CELLERIER, Jean Fernand } \\
(1870-1936)\end{array}$ & $\begin{array}{l}\text { "Choix et classification des caractéristiques scientifiques d'une œuvre } \\
\text { d'art en vue de l'identification et la conservation" } \\
14 \text { Octobre - Martin Section A. Peinture }\end{array}$ & $\begin{array}{l}\text { Laboratoire d'Essais et d'Identification des Peintures et } \\
\text { des CEuvres d'Art du Musée du Louvre; } \\
\text { Laboratoire d'Essais du Conservatoire National des Arts } \\
\text { et Métiers à Paris. (1928-30) }\end{array}$ \\
\hline $\begin{array}{l}\text { 4. CHAMPION, Benoît } \\
\text { Claude (1863-1952) }\end{array}$ & $\begin{array}{l}\text { "De la nécessité d'une technique d'observation sous forme d'analyses } \\
\text { descriptives, pour I' identification des objectes archéologiques» } \\
14 \text { Octobre - Martin Section B. Sculpture }\end{array}$ & $\begin{array}{l}\text { Atelier de moulage et de galvanoplastie du Musée des } \\
\text { Antiquités Nationales de Saint-Germain-en-Laye (1866). } \\
\text { Atelier Technique (1927) }\end{array}$ \\
\hline $\begin{array}{l}\text { 5. CHAMPION, Pierre (1902- } \\
\text { 1978) }\end{array}$ & $\begin{array}{l}\text { "Equipement de bais et verrières en verres spéciaux absorbant les } \\
\text { radiations ultra-violettes et atténuant l'altération de la teinte des objets } \\
\text { exposés au rayonnement solaire» } \\
16 \text { Octobre - Jeudi Section Planaire }\end{array}$ & Laboratoire du Musée d'Ethnographie à Paris (1882) \\
\hline $\begin{array}{l}\text { 6. CHIERICI, Gino (1877- } \\
\text { 1961) }\end{array}$ & $\begin{array}{l}\text { "De la nécessité de publier une Revue des restaurations d'ouvres d'art" } \\
17 \text { Octobre - Vernerdi } \\
\text { Section Plenaire }\end{array}$ & LaboratoireduNaples (1925) \\
\hline $\begin{array}{l}\text { 7. CIALDEA, Umberto (1908 } \\
\text { - 1940) }\end{array}$ & $\begin{array}{l}\text { «Restaurationdes bronzes anciens» } \\
15 \text { Octobre - Mardi Section B. Sculpture }\end{array}$ & $\begin{array}{l}\text { Laboratoire du Palatin - École pratique de Physique et } \\
\text { Physico-chimie à I'Université Royale de Rome (1930) }\end{array}$ \\
\hline $\begin{array}{l}\text { 8. EIBNER, Alexander } \\
\text { (1862-1935); } \\
\text { GRÄEFF, Walter (1876- } \\
\text { 1934) }\end{array}$ & $\begin{array}{l}\text { "L'état actuel de l'examen micro chimique des peintures» } \\
13 \text { Octobre - Lundi Section A. Peinture }\end{array}$ & $\begin{array}{l}\text { Versuchsanstalt und Auskunftsstelle für Maltechnik } \\
\text { (1902-1935) } \\
\text { Laboratoire Radiographique (1916-1934) }\end{array}$ \\
\hline $\begin{array}{l}\text { 9. GOULINAT, Jean-Gabriel } \\
\text { (1883-1972) }\end{array}$ & $\begin{array}{l}\text { "Quel parti le technicien de la peinture» } \\
15 \text { Octobre - Mardi } \\
\text { Section A. Peinture }\end{array}$ & $\begin{array}{l}\text { Laboratoire d'essais des Arts et métiers, Louvre, Paris } \\
\text { (1928) }\end{array}$ \\
\hline $\begin{array}{l}\text { 10. KÖGEL, Gustav (1882- } \\
\text { 1945) }\end{array}$ & $\begin{array}{l}\text { "L'identification des œuvres d'art et l'examen photographique» } \\
14 \text { Octobre - Martin Section A. Peinture }\end{array}$ & $\begin{array}{l}\text { University of Karlsruhe - Institute for Technical } \\
\text { Photochemistry and Scientific Photography (1921) }\end{array}$ \\
\hline $\begin{array}{l}\text { 11. LAURIE, Arthur Pillans } \\
\text { (1861-1949) }\end{array}$ & $\begin{array}{l}\text { "1) L'application des agrandissements photographiques à l'examen de la } \\
\text { technique des peintures; } 2 \text { ) REMBRANDT ET SON Ecole; expérience sur sa } \\
\text { technique personnelle. L'influence de Rembrandt sur ses élèves.» } \\
14 \text { Octobre - Martin Section A. Peinture }\end{array}$ & $\begin{array}{l}\text { Department of Scientific and Industrial Research - Royal } \\
\text { Academy of Arts (UK) (1923) }\end{array}$ \\
\hline 12. LEVI, Alda (1890-1950) & $\begin{array}{l}\text { "L'analyse chimique des terres cuites anciennes en vue de l'identification } \\
\text { de leur provenance» } \\
14 \text { Octobre - Martin Section B. Sculpture }\end{array}$ & Laboratoire du Institut Polytechnique de Milan (1925) \\
\hline $\begin{array}{l}\text { 13. MERCIER, Fernand } \\
\text { (1884-1986); } \\
\text { ESCHOLIER, Raymond } \\
\text { (1882-1971) }\end{array}$ & $\begin{array}{l}\text { "De la necéssité de créer un centre de documentation scentifique et } \\
\text { artistique» } \\
17 \text { Octobre - Vernerdi } \\
\text { Section Plenaire }\end{array}$ & $\begin{array}{l}\text { Laboratoire de microradiographie, Musée des Beaux } \\
\text { Arts de Dijon (1922) }\end{array}$ \\
\hline $\begin{array}{l}\text { 14. RINNEBACH, Helmuth } \\
\text { (1870-1942) }\end{array}$ & $\begin{array}{l}\text { «L'examen comparatif des peintures aux Rayons X. Son importance et ses } \\
\text { limites» } \\
14 \text { Octobre - Martin Section A. Peinture: }\end{array}$ & $\begin{array}{l}\text { Deutsches Bilderarchiv (Archives allemandes des table) } \\
\text { - Laboratoire Central d'Études des Tableaux - Berlin (?) }\end{array}$ \\
\hline $\begin{array}{l}\text { 15. RORIMER, James (1905- } \\
\text { 1966) }\end{array}$ & $\begin{array}{l}\text { "L'application des Rayons ultra violets à l'examen des sculptures en } \\
\text { marbles» } \\
13 \text { Octobre - Mardi Section B. Sculpture }\end{array}$ & MetropolitanMuseumofArt (1927) \\
\hline $\begin{array}{l}\text { 16. SCHEFFER, F.E.C. (1882- } \\
\text { 1958); } \\
\text { WILD, Angenitus Martinus } \\
\text { de (1899-1969) }\end{array}$ & $\begin{array}{l}\text { "L'examen chimique des tableaux» } \\
13 \text { Octobre - Lundi Section A. Peinture }\end{array}$ & Laboratoire de l'Université Technique de Delft (1928) \\
\hline $\begin{array}{l}\text { 17. STOUT, George Leslie } \\
\text { (1897-1978) }\end{array}$ & $\begin{array}{l}\text { "Expériences effectuées en vue de la conservation des peintures murales } \\
\text { asiatiques au moyen d'un transport sur nouveaux supports» } \\
15 \text { Octobre - Mardi Section A. Peinture }\end{array}$ & $\begin{array}{l}\text { Department for Technical Studies (1925) Fogg Museum, } \\
\text { Harvard University }\end{array}$ \\
\hline 18. VITRY, Paul (1872-1941) & $\begin{array}{l}\text { «La conservation des sculptures et le moulage» } \\
15 \text { Octobre - Mardi Section B. Sculpture }\end{array}$ & $\begin{array}{l}\text { Laboratoire des recherches scientifiques - Paris (1928- } \\
\text { 30) }\end{array}$ \\
\hline $\begin{array}{l}\text { 19. WILDE, Johannes (1891- } \\
\text { 1970) }\end{array}$ & $\begin{array}{l}\text { "Présentation des photographies obtenus aux Rayons Röntgen de } \\
\text { quelques tableaux exécutés à l'Institut Holzknecht à Vienne» } \\
14 \text { Octobre - Martin Section A. Peinture }\end{array}$ & X-Ray Laboratory Kunsthistorisches Museum (1915) \\
\hline
\end{tabular}


As profundas transformações urbanas, por meio de planos, reformas e remodelações - como o projeto de Georges-Eugène Haussmann (1809-1891) para Paris, implantado entre 1853 e 1870; o Plan Cerdá para Barcelona em 1860; a Reforma Pereira Passos entre 1902 e 1906 para a cidade do Rio de Janeiro - e as destruições causadas pelas guerras e conflitos sociais em monumentos e edifícios históricos determinaram o nascimento simultâneo da teoria e da prática da conservação-restauração arquitetônica e de bens integrados ao edifício. Por sua vez, as demandas de organização das coleções dos museus públicos, das bibliotecas e dos arquivos também foram determinantes na configuração de um novo ator social capaz de associar os princípios histórico-filosóficos aos métodos de gestão desses acervos. Nesse contexto, moldado pela Revolução Industrial e pelas guerras Napoleônicas, despontam os primeiros teóricos da conservação no campo da arquitetura: John Ruskin (1819-1900), com "The Seven Lamps of Architecture", de 1849; Eugène Emmanuel Viollet-le-Duc (1814-1879), com "Dictionnaire Raisonné de l'Architecture Française du XI au XVI Siècle", de 1868 e Camillo Boito (18361914), promotor do " $3^{\circ}$ Congresso degli Ingegneri e Architetti italiani" de Roma em 1883, o qual gerou a "Carta Italiana del Restauro". Derivam dessas bases os principais teóricos do início do século XX, como Gustavo Giovannoni (1873-1947) e Luca Beltrami (1854-1933) (CASTRIOTA, 2008).

A Escola de Viena também produziu uma sistematização do pensamento no campo do patrimônio, uma vez que seus principais teóricos combinaram suas carreiras acadêmicas com atividades curatoriais em museus e a gestão de monumentos. A obra "Der moderne Denkmalkultus" de 1903 de Alois Riegl (1858-1905) foi forjada sob a demanda de organização da Comissão de Monumentos Históricos da Áustria, assim como Max Dvořák (1874-1921) publica Catecismo da Preservação de Monumentos em 1916 a partir de sua experiência em gestão pública dos monumentos austríacos (SCHAPIRO, 1936).

Contudo, foram os anos seguintes à Primeira Guerra Mundial (1914-1918) que definiram indubitavelmente as alterações nos protocolos da conservação-restauração, a introdução de uma metodologia científica de análise e o estabelecimento de normas de proteção de bens culturais (PLENDERLEITH, 1998). As primeiras conferências, laboratórios e publicações foram estruturados nesse contexto, assim como a discussão dos primeiros marcos legais do direito internacional (FOUNDOKIDIS, 1939), o que nos leva a situar neste momento histórico a projeção de uma visão ampliada no campo da cultura e da proteção do patrimônio.
Em 04 de janeiro de 1922, a Liga das Nações, fundada em 28 de junho de 1919, estabeleceu as bases da cooperação internacional por meio da criação do Comitê Internacional para a Cooperação Intelectual, o qual atuará até 1946. Suportado pela representatividade diplomática dos países membros, questões concernentes ao intercâmbio de pesquisa e formação foram debatidas por este conselho. Em 24 de julho de 1924, o governo francês apresentou à Liga a oferta de estabelecimento de uma sede em Paris com o intuito de suportar os trabalhos do Comitê e em dezembro do mesmo ano, na Assembleia Geral, a proposta de criação do Instituto Internacional para a Cooperação Internacional foi aprovada, sendo inaugurada em 16 de janeiro de 1926 no Palais Royal de Paris (LEVEAU, 2014).

Considerando as necessidades de restauro, repatriamento de espólios e atuação coletiva no âmbito da proteção do patrimônio cultural, sob a iniciativa do Comitê Internacional de Cooperação Intelectual, em julho de 1926 foi criado o Escritório Internacional de Museus. Sob os auspícios do Instituto, o Escritório produziu uma das mais importantes publicações da área, a revista "Mouseion" (CAILLOT, 2011), além de organizar encontros internacionais e, ao longo dos anos trinta, efetuar um estudo que resultaria em um projeto de elaboração de uma "Convenção para a Proteção de Monumentos e Obras de Arte em tempos de guerra" (1939).

Apesar da saída do Brasil da Liga das Nações em 1926, o Comitê Nacional de Cooperação Intelectual, fundado em 1923, permaneceu ativo até sua extinção. Em distintos momentos, seus membros se posicionaram em prol da cooperação internacional por meio das comissões nacionais. Nas palavras de posse como presidente da comissão brasileira, Miguel Ozório de Almeida (1890-1952) afirma: “Não tenhamos receio de repetir: a ciência, para o seu progresso, depende da qualidade de alguns indivíduos criadores, mas em seu conjunto é trabalho coletivo e universal, e não é com o desejo de isolamento que se poderá colaborar em trabalhos de tal natureza" (ALMEIDA, 1939, p.938). O intelectual do início do século XX emerge sob o crivo de um pensamento crítico. No contexto entreguerras não há mais lugar para um distanciamento positivista, e o mito da neutralidade científica sucumbe diante de um novo ator que insurge sob um fundo cultural e sob uma forma de papel político-social (MORIN, 1983).

\section{A CONFERÊNCIA DE ROMA COMO MARCO HISTÓRICO: A MUDANÇA DE PARADIGMA}

Dentre as ações conjuntas do Instituto Internacional de Cooperação Internacional, do Escritório Internacional de Museus e das comissões 
nacionais para cooperação intelectual, o marco fundamental para o campo científico da Conservação-Restauração é, sem dúvida, a Conferência Internacional para o Estudo dos Métodos Científicos aplicados ao Exame e à Conservação de obras de Arte que ocorreu entre 13 e 17 de outubro de 1930, em Roma. A partir deste encontro é possível demarcar a integração gradual da ciência no âmbito museológico e da gestão pública dos monumentos, bem como o estabelecimento da colaboração entre o conservador-restaurador, o cientista e o curador. Ressalta-se que o princípio colaborativo entre esses atores nem sempre é linear, ausente de confrontos ou assente em relação à prerrogativa das ações.

No relatório da reunião executiva do Escritório Internacional de Museus que ocorreu nos dias 30 e 31 de janeiro de 1930, pela primeira vez se menciona a proposição do encontro, abordada no sétimo ponto da pauta:

Le Bureau accueille avec sympathie la proposition formulée par M.A, Rossi, au nom de la Commission italienne de Coopération intellectuelle de tenir à Rome, dans la première semaine d'octobre, la conférence d'experts pour la conservation, la restauration el l'examen scientifique des œuvres d'art, et charge le Secrétariat de préciser un plan pour la réunion du Bureau, au mois de mai prochain" (OIM, 1930, p.77-81).

A carta convite, datada de 19 de setembro de 1930, com cópia ao Conde Francesco Pellati(18821967) - Direttore Capo de Division e presso La Direzione Generale per Antichit'a e Belle Arti -, evidencia o envolvimento do governo italiano, do Escritório de Museus e da Comissão Nacional Italiana na promoção do evento. Por meio desta articulação política, em carta datada de 20 de novembro de 1930, Atílio Rossi (1909-1994) foi nomeado membro da Comissão de Museus do Escritório, atuando também como secretário geral do Instituto de Cooperação Internacional.

O empenho de Rossi para a realização do evento pode ser mapeado por meio dos registros L'Activite de l'Office International dês Musées, publicados na Museion. No volume 11 de 1930, a sétima pauta da reunião dos dias 29 e 30 de maio de 1930 descreve:

Le Bureau a entendu successivement les communications présentées par le Secrétariat de I'Office International des Musées et par M. Attilio Rossi, au nom de la Commission italienne de coopération intellectuelle.ll a exprimé ses remerciements à la Commission italienne et, après discussion, a décidé : 10 Que la réunion d'experts pour l'étude des problèmes relatifs à l'examen scientifique et la conservations des œuvres d'art aura lieu à Rome, du 13 a 17 d'octobre ; 20 Cette conférence comprendra deux sections, une consacrée à la peinture, l'autre à la sculpture ; 3응
Les travaux de la conférence serons organisés suivant un plan dont il a arrêté les dispositions générales ; 4 ㅇ II a donné mandant au secrétaire de I'OIM de mettre au point la préparation de cette réunion par des rapports directs avec la Commission italienne de coopération intellectuelle. Le président du Bureau de l'Office sera consulté pour le règlement de toutes les questions qui n'auraient pas été prévues par le Bureau(OIM, 1930, p.184-210).

O encontro reuniu cento e quarenta e dois participantes de vinte dois países, sendo que de Portugal foi representado por José de Figueiredo (1972-1937), como Diretor do Museu Nacional de Arte Antiga de Lisboa, e Luciano Freire (1864-1934), como Presidente do Conselho de Arte e de Arqueologia de Lisboa. Apesar da atuação contemporânea ao evento de Roberto de Carvalho (1893-1944) e de Pedro Vitorino (1882-1944) no campo da radiografia aplicada ao estudo da obra de arte em Portugal, estes pesquisadores portugueses não participaram do evento.

O conceito de "experts" na conferência abarcou químicos, físicos, arqueólogos, historiadores da arte, conservadores-restauradores e curadores de museus, entre outros profissionais dedicados ao estudo, gestão e conservação-restauração de bens culturais.

Com cinquenta e sete conferências proferidas por quarenta e oito pesquisadores, os primeiros laboratórios instalados em museus e universidades puderam divulgar suas pesquisas. O levantamento dos laboratórios na Tabela 1 foi possível graças aos documentos encontrados no Fondo Brizzio, Fondo Pellati, às comunicações apresentadas na Mouseion e ao estudo de Cardinali e Ruggie ripublicado em "Snodidi Critica" (2013). A maioria deles teve suas atividades interrompidas por causa da Segunda Guerra Mundial (1939-1945), mas alguns retomaram suas atividades após o conflito, permanecendo ativos ainda hoje.

Como resultado imediato do encontro, é possível observar que as publicações da década de trinta da revista "Museion" maioritariamente foram direcionadas aos estudos das condições ambientais nos museus; exames analíticos de obras de arte e monumentos; métodos e técnicas relacionadas à conservação-restauração de bens culturais; além de distintos resultados de pesquisas e trabalhos desenvolvidos em obras, coleções, museus, sítios e monumentos. Três sessões apresentam debates de especial interesse no campo historiográfico e epistemológico da Ciência da Conservação: os registos "L'Activité de l'Office International dês Musées", que indicam os encaminhamentos políticos e as áreas de interesse das respectivas direções; a sessão "La conservation de œuvres 
d'art", eventualmente direcionada ao debate crítico em torno dos marcos legais, da regulamentação da profissão do conservador-restaurador e de projetos colaborativos entre áreas; e a sessão "Muséographietechnique", que antecipa o conceito de Conservação Preventiva.

Os suplementos em Inglês, Alemão, Italiano e Espanhol, publicados na revista acerca das conclusões adotadas na Conferência Internacional para o Estudo dos Métodos Científicos Aplicados ao Exame e Conservação de Obras de Arte, apresentam o impacto da visão científica da "curadoria" e do restauro dos acervos (OIM, 1931). Nas recomendações são levantados os seguintes itens: I. Demanda de planeamento do espaço dos museus para gerar "condições mais favoráveis" para a conservação; II. Exames científicos preliminares, antes da limpeza e do restauro, bem como a documentação fotográfica e gráfica dos registros do processo de intervenção; III- O emprego dos vernizes de proteção; IV- A solicitação ao Escritório Internacional de Museus a redação de um Manual de Conservação e Restauração de Pinturas a ser distribuído aos Diretores de Museus. Recomenda-se também a publicação das conferências, o que ocorrerá de fato nas edições de 1931 e 1932 da revista.

O I Congresso Internacional de Arquitetos e Técnicos em Monumentos de 1931, que produziu um dos mais importantes documentos da área (a Carta de Atenas) e promoveu a criação da Comissão Internacional de Monumentos Históricos (CIMH), ocorreu como resultado direto da Conferência de Roma.

No relatório apresentado à XIII Sessão do Comitê Internacional de Cooperação Intelectual, Jules Destrée (1863-1936), presidente do Escritório Internacional de Museus, comenta:

La principale activité de l'Office, dans le domaine de la conservation de œuvres d'art, a porté sur l'organisation de la Conférence internationale d'experts qui s'est tenue à Rome en octobre de 1930. [...] Le programme de Rome a dû cependant être limité aux seules œuvres de peinture et de sculpture. Aussi, pour répondre aux nombreuses sollicitations qui luit ont été adressés depuis la Conférence de Rome, le Comité de direction de l'Office s'est vu amené à envisager sans retard la convocation d'une nouvelle conférence. Suite logique et nécessaire du programme de Rome, elle sera consacre cette fois aux problèmes relatifs à la protection et à la conservation des monuments $d^{\prime}$ 'art et d'histoire et viendra compléter le cycle des problèmes de conservation ressortissant aux trois grandes divisions des arts plastiques: peintures, sculptures, architecture, dont l'étude est prévue par les statuts de l'Office. Grâce à la bienveillance du Gouvernement hellénique, la conférence pourra se tenir à Athènes, cadre particulièrement approprié, qui ajoutera encore à la portée et à l'intérêt de nos travaux (DESTRÉE, 1931, p.91-106).

As conferencias seguintes, em Madrid (1934), sobre "Architecture et aménagement des musées", e Cairo (1937), "Conference Internationale dês Fouilles", resultam diretamente das discussões propostas nos dois primeiros eventos. Todos estes encontros determinaram os campos de estudos da Ciência da Conservação e da Conservação Preventiva, produzindo as primeiras publicações na área no âmbito internacional e demarcando o princípio colaborativo entre os laboratórios e os pesquisadores.

O "Manuel de La Conservation et de La Restauration dês Peintures" foi editado pela primeira vez em 1938 nos volumes 41-42 da Revista Mouseion, e posteriormente publicado pelo Escritório Internacional de Museus sob a organização de Constable em 1939. O conteúdo produzido de forma coletiva foi definido a partir de um encontro de especialistas promovido pelo Escritório em Paris, em 1933, sob a presidência de W.G. Constable (1905-1981), diretor do Courtauld Institute, conforme as deliberações do Encontro de Roma. Nessa reunião estiveram presentes os principais cientistas, conservadores e pesquisadores da área do patrimônio: Baud-Bovy (1870-1958), Folch y Torres (1880-1950), Paul Ganz (1872-1956), Goulinat (1883-1972), Laurie (1861-1949), E. Lavagnino (1898-1963), J. Maroger (1884-1962), A. Martin de Wild (1891-1970), Helmut Ruhemann (1891-1973), George L. Stout (1897-1978) e Plenderleith (1898-1997), os quais foram responsáveis pela organização e redação dos conteúdos. A referência desses nomes aparece no "Butlletídels Museus d'Art" de Barcelona e também no elenco do comitê de redação da edição especial "La Conservation dês Peinturas" da Mouseion (1938), ainda que usualmente a redação do manual seja atribuída apenas à Stout, Ruhemann e Plenderleith.

Além do manual de pinturas, outras publicações relacionadas às conferencias estruturaram modelos de pesquisa, produção de artigos e organização de encontros, conformando o campo epistemológico da área: "La Conservationdesmonuments d'art et d'histoire", de 1933; "Museographie: architecture et aménagement dês musées d'art", de 1935 e "Manuel de latechnique des fouilles archéologiques", de 1939, são as primeiras e mais importantes publicações internacionais que de fato discutiram o alcance da cooperação intelectual no campo da ciência e do patrimônio. 


\subsection{A defesa do patrimônio artístico e a formação do conservador-restaurador}

As conferências citadas foram decisivas para lidar de forma subsidiada com as questões de difusão, conservação-restauração e pesquisa de bens culturais. A partir de então, questões primordiais foram colocadas à área: criação de laboratórios para o desenvolvimento de estudos de bens culturais e métodos de intervenção; compartilhamento de pesquisas, formação de pessoal qualificado e proteção legal do patrimônio. Sob esta perspectiva, quatro artigos publicados imediatamente após os encontros apontam de forma cabal as relações intrincadas da formação como condição sinequa non à Preservação do Patrimônio Cultural no século XX.

O primeiro, "Pour une éducation professionnelle des restaurateurs d'oeuvres d'art" (1932), assinado por Dr. Robert Eigenberger (1890-1979) e um grupo de especialistas vinculados à Universidade e ao Escritório dos Monumentos de Viena, imprime uma discussão extremamente complexa sobre reconhecimento profissional e formação, rompendo com conceitos vinculados à disposição de uma prática amadora, dispensável de pesquisa ou de responsabilidade social. $\mathrm{O}$ texto afirma:

\begin{abstract}
La conservation des œuvres d'art pose des problèmes qui préoccupent de plus en plus les milieux intéressés. Les deux conférences organisées par les soins de l'Office International des Musées, à Rome, puis à Athéna, en 1930 et 1931, répondaient précisément au souci de voir ces questions sortir de l'empirisme et appeler l'attention des directeurs de musées sur l'apport considérable que la science moderne leur offre au jour hui. Les suggestions qu'on trouvera cidessous, et qu'un groupe de personnalités autrichiennes vient d'adresser à l'OIM, sont conformes à ces préoccupations et pourraient être le point de départ d'une organisation rationnelle dans la technique de la conservation des œuvres d'art [...] Parmi les causes de destruction qui menacent les œuvres d'art, les interventions de restaurateurs incompétents sont les plus dangereuses [...] (p.83-85).
\end{abstract}

A questão da competência aqui abalizada não se refere à habilidade técnica ou artística, mas à competência no que tange à capacidade e à autoridade conferida pela investidura de uma formação subsidiada por um determinado campo de saber. A demanda da constituição de um lócus exemplar de repertório, conhecimento, pesquisa e compartilhamento de ideias.

O "mundo misterioso dos museus", repleto de "fórmulas mágicas" e "segredos de profissão", não responderia mais às demandas reais de restauração de bens culturais, organização dos espaços museais e das medidas legais em torno da proteção do patrimônio cultural, principalmente após a Primeira
Guerra Mundial. As profundas alterações sociais e a expansão da comunicação, por meio do rádio e do cinema, tornaram públicas as mais importantes coleções, sítios e monumentos em diversos países. Ao problema anunciado sobre a demanda de profissionalização, as proposições apresentadas são sistematizadas da seguinte forma: 10 criação de centros de instrução para formação de restauradores, preferencialmente vinculados às instituições de Belas Artes; 20 Criação de espaços de instrução compostos por laboratórios técnicos auxiliares (química, radiografia, etc.); 3ํㅡㄹ Capacitação certificada por um diploma.

No texto de Eigenbergeraparece o termo "ciência da conservação": "Point n'est besoin d'insister sur les graves inconvénients que comporte, pour la science de la conservation, l'absence de toute institution analogue" (1932, p. 83-85).Este termo, presente no discurso proferido no fechamento da Conferência de Roma em 1930 por Jules Destrée, conforme documentação encontrada no Fondo Francesco Pellati, torna-se fundamental à discussão acerca da atividade científica relacionada à preservação de bens culturais materiais.

A proposta apresentada prevê ainda que a profissão de restaurador deveria ser sujeita à licença especial, considerando a experiência prévia de muitos especialistas. Contudo, o exercício da profissão sem regulamentação não deveria prosseguir. Uma das questões postas que aproximam das recomendações "ECCO Professional Guidelines", mais de setenta anos depois refere-se à formação: esta deveria inicialmente ser geral e cobrir todas as técnicas e, sobretudo, todos os materiais. A especialização deveria ser adquirida apenas posteriormente, visando a formação dos restauradores especialistas em determinados domínios particulares. A percepção da construção de um percurso formativo progressivo antecipa as discussões atuais da área. De acordo com Figueira (2015),

Desde 2004 que a ECCO Professional Guidelines III reconhece o grau de mestrado precedido de licenciatura em conservação-restauro $(3+2$ anos) como exigência mínima para a entrada na profissão. No entanto, a reforma iniciada pelo Processo de Bolonha, em 2006, veio confundir e alterar esse acesso à profissão lançando no mercado profissionais com apenas 2 anos de mestrado em conservação-restauro precedido de licenciatura noutra área de formação ou profissionais com apenas 3 anos de licenciatura.

Se por um lado a atuação profissional parece fragilizada, por outro lado a área amplia o acesso ao desenvolvimento de pesquisa por meio da inclusão de outros campos de saber, como a Química, a Física, a Geologia e a Biologia. 
Na mesma edição em que aparece o texto de Eigenbergerem 1932, Victor Bauer-Bolton escreve o artigo"Pour une réglementation juridique de la conservation des œuvres d'art", no qualafirma: "En d'autres termes, si les commissions techniques doivent se constituer selon les différents arts, les dispositions légales à prendre pour la protection des chefs-d'œuvre doivent être les mêmes pour tous" (p.148-149).

Os artigos de Bauer-Bolton e Eigenberger parecem provocar uma movimentação em torno do tema. Em 1932, Robert Maurer e HelmuthRinnebach(1870-1942)publicam na seção"La conservation de oeuvres d'art"o texto "La défense du patrimoine artistique et la formation des restaurateurs". Nas primeiras páginas escritas por Maurer, duas questões cruciais são abordadas sob o prisma da necessidade de preservação do patrimônio cultural no pós-guerra: a evasão de bens culturais e a ação indiscriminada na intervenção em objetos e monumentos. No primeiro caso, aponta as relações legais do Estado, e no segundo, tomando como premissa o contexto francês, recomenda a criação de uma comissão superior, indicada pelo Ministério de Instrução Pública em acordo com as organizações profissionais, composta por historiadores da arte, pintores, colecionadores e restauradores, com o intuito de estabelecer as bases legais para a atuação na área. Rinnebach, por sua vez, introduz ao debate a demanda de uma transição gradual no exercício da profissão; aponta o conhecimento de técnicas e materiais, além do conhecimento geral em química e físico-química como princípios fundamentais à avaliação da expertise; ainda solicita que as comissões formadas tenham autoridade para encaminhar aos tribunais "as infrações graves no exercício da profissão" passíveis de sanções penais.

Em 1933, dando sequência ao debate, Wilhelm Waetzoldt (1880-1940), diretor geral do Museu do Estado de Berlim, e Richard Graul (1862-1944), exdiretor do Museu de Leipzig, apresentam o texto "La défense Du patrimoine artistique et La formation dês restaurateurs", mesmo título utilizado no artigo anterior. Os autores colocam que simultaneamente aos processos de regulamentação da profissão, seria fundamental a criação de novos estabelecimentos de ensino voltados à formação do conservadorrestaurador e, associados a eles, a colaboração técnica de laboratórios e instituições já estabelecidas. A certificação gerenciada por meio dos órgãos públicos estatais deveria organizar a formação a partir das diretrizes dos sistemas educacionais vigentes; regulamentar o exercício profissional e prever, considerando a prática já sistematizada, um sistema de incorporação de conservadores-restauradores já atuantes

\section{CONSIDERAÇÕES FINAIS}

As discussões propostas na primeira metade do século XX não puderam avançar em função do conflito bélico subsequente. No entanto, o aporte e a projeção internacional da Carta de Atenas, uma das primeiras recomendações internacionais; as conferência e publicações promovidas pelo Escritório Internacional de Museus; os documentos que marcam a discussão dos aspectos legais de proteção no âmbito internacional e a criação de diversos laboratórios na área da Ciência da Conservação devem ser contemplados à luz das transformações do paradigma contemporâneo da Ciência do Patrimônio e fazem parte de uma temporalidade ampliada que comporta todo o século XX.

Os resultados imediatos desta movimentação em torno do Estado da Arte da Preservação foram significativos e posteriormente retomados por instituições como o ICOM, o ICCROM e o ICOMOS sob os auspícios da UNESCO.

As discussões estruturadas no período entreguerras - formação, ciência e acordos legais - são questões atualizadas e exigem uma percepção engajada da atuação do pesquisador na área da Ciência do Patrimônio em relação às situações emergentes, como a demanda de construção solidária do conceito de cultura e de ciência; o compartilhamento das pesquisas por meio de redes colaborativas; e a efetiva proteção de bens culturais ancorada em uma percepção inter-politransdisciplinar.

A epistemologia que demarca a teoria do conhecimento e as relações históricas de construção do campo do saber da Ciência da Conservação compõem o quadro atual de revisões historiográficas no que concerne à Ciência do Patrimônio, fortalecendo a própria apreensão da área. Diante da pluralidade dos problemas vivenciados pelas sociedades contemporâneas,este ramo científico se estabelece a partir da potencialização e do compartilhamento de áreas do conhecimento voltadas aos estudos de bens culturais.

A Ciência do Patrimônio busca investigar, preservar e conservar o patrimônio cultural no seu sentido expandido (material e imaterial), utilizando métodos oriundos das Ciências Humanas e das Ciências Naturais. Consequentemente, diferentes campos do conhecimento, como a Ciência da Informação, a Ciência da Conservação, a Conservação Preventiva, a História da Arte, as Ciências Sociais e as Ciências Jurídicas, quando aplicados ao patrimônio cultural, produzem um campo transdisciplinar sinérgico capaz de responder aos estudos culturais a partir de uma visão ampliada. 
Portanto, questões econômicas e sociais, como Sustentabilidade e Resiliência, também podem (e devem) ser incorporadas a este debate, bem como os estudos históricos sobre a origem e os fundamentos filosóficos da Ciência Patrimonial. Se situa no debate em torno do "pensamento complexo" da Ciência e da Educação contemporânea estabelecido por Edgar Morin, com a fundação do Centre International de Recherches et études Transdisciplinaires (CIRET), em 1987.

Todas as questões parecem extremamente prementes, principalmente no contexto brasileiro de regulamentação da profissão e de estruturação científica das áreas e linhas de pesquisa nas agências de fomento.

\section{AGRADECIMENTOS}

Agradeço à CAPES pela subvenção desta pesquisa de estágio sênior em Ciência do Patrimônio (2015-2016). À arquivista do ICCROM, Maria Matta Caravaca; à arquivista da Accademia Nazionale dei Lincei, Paola Cagiano de Azevedo e à arquivista Cristina Selva da Biblioteca di Storia dell'Arte, della Musica e dello Spettacolo da Università degli Studi di Milano. Às equipes da Biblioteca do ICCROM e da Biblioteca di Archeologia e Storia dell'Arte. Agradeço especialmente ao Diretor-geral do ICCROM, Dr. Stefano De Caro, pelo apoio institucional à pesquisa e ao Programa e Pós-graduação em Ambiente Construído e Patrimônio Sustentável da UFMG pelo apoio à participação neste evento.

\section{REFERÊNCIAS BIBLIOGRÁFICAS}

'Carta convite do Ministro Guardasigilli (19/09/1930)', cópiadatilografada, Biblioteca dell'Accademia dei Lincei e Corsiniana, Fondo Francesco Pellati, Caixa 6, 171, 1930.

'Carta de nomeação de Rossi (20/11/1930)', cópiadatilografada, Biblioteca dell'Accademia dei Lincei e Corsiniana, Fondo Francesco Pellati, Caixa 6, 171, 1930.

'Conferenza internazionale di Roma per lo studio dei metodi scientifici applicati all'esame e alla conservazione delle opere d'arte, Roma 13-17 ott. 1930: corrispondenza, programma, o.d.g., delegazione italiana, relazioni', cópiadatilografada, Biblioteca dell'Accademia dei Lincei e Corsiniana, Fondo Francesco Pellati, Caixa 6, 171, 1930.

ALMEIDA, M.O. de. Solidariedade internacional - missão do intelectual brasileiro, conferência no Itamaraty sob os auspícios do Comitê Brasileiro de Cooperação Intelectual. In: Mensário do Jornal do Comércio III (1), Rio de Janeiro: MJC, 1939, p.938-940.

ANTI, C.. et al..Manuel de la technique des fouilles archéologiques.Paris: International Institute of Intellectual Co-operation-Office International des Musées, 1935.

BALLESTREM, Agnes. The Conservator-Restorer: a definition of the profession, Rome: ICCROM Standards and Training Committee, 1978.

BAUER-BOLTON, V. Pour une réglementation juridique de La conservation des œuvres d'art, Mouseion, 19 (3), 1932, Paris: OIM, p.148-149.
BMAB, Una reuniéa Paris de La Comisseió d'experts per la conservació de pintures, convocadapel I'Office International des Musées, ButlletídelsMuseus d'Art de Barcelona, 3, 1933, p.212-216.

CAILLOT, M., La revue Mouseion (1927-1946) - Les musées et la coopération culturelle international, Paris: Université de Sorbonne, 2011.

CARDINALI, M; RUGGIERI, M. 'El pensiero critico e le richerche tecniche sulle opera d'arte a partir dalla conferenza di Roma del 1930, in Snodi di critica: musei, mostre, restauro e diagnostica artistica in Italia 1930-1940, ed. M. I. Catalano, Roma: Armando Editore, 2013, p. 107-150.

CASTRIOTA, Leonardo. Patrimônio Cultural: conceitos, políticas, instrumentos, São Paulo: Annablume, 2009.

CATALANO, M.I. Snodi di critica: musei, mostre, restauro e diagnostica artistica in Italia 1930-1940, Roma: Armando Editore, 2013.

CLARK, Burton R., The Research Foundations of Graduate Education: Germany, Britain, France, United States, Japan, Los Angeles: University of California Press, 1993.

Commissione Nazionale Italiana per La Cooperazione Intelletualle. 'Conférence internationale pour l'étude des méthodes scientifiques appliquées à l'examen et à la conservation des œuvres d'art - Membres Participants', cópiadatilografada, FondoBriziodell'UniversitàdegliStudi di Milano, 1930.

CONSTABLE, W.G. et al..Manuel de la Conservation et de la Restauration des Peintures, Paris: International Institute of Intellectual Co-operation-Office International des Musées, Paris, 1939.

CRUZ, A. J. O início da radiografia de obras de arte em Portugal e a relação entre a radiografia, a conservação e a política, Conservar Património 11, 2010,p.13-32, http://ciarte.no.sapo.pt/textos/fichas/201003.html. (acesso em 2016-03-21)

DESTREE, J.'L'Activite de l'Office International des Musées, Mouseion, 15 (3), 1931, p.91-106.

DESTREE, J.. et al., La Conservation des monuments d'art et d'histoire, International Institute of Intellectual Co-operationOffice International des Musées, Paris (1933).

ECCO. Competences for access to the conservation-restoration profession, European Confederation of ConservatorRestorers' Organisations, http://www.ecco-eu (acesso em 2015-10-10)

EIGENBERG, R. et al. Pour une éducation professionnelle des restaurateurs d'oeuvres d'art, Mouseion, 19 (3), 1932, p.8385.

ENCoRE. On Practice in Conservation-Restoration Education, European Network of Conservation-Restoration Education, http://www.encore-edu.org/ (acesso em 2015-10-10)

FIGUEIRA, F.A disciplina/profissão de conservação-restauro: uma ciência recente e seu desenvolvimento em Portugal, Conservar Património 21, 2015, p. 39-51, doi:10.14568/cp2014004, http://revista.arp.org.pt/pdf/2014004.pdf (acesso em 201610-10).

FOUNDOUKIDIS, $E$ et ali.. La Conservation des Peintures, Mouseion, 41-42 (1-2), 1938.

FOUNDOUKIDIS, E. La Protection des Monuments et CEuvres d'Art Temps de Guerre, Mouseion, 47-48 (3-4), 1939.

FRONER, Y.A. Os domínios da memória, tese, Universidade de São Paulo, São Paulo, (2001). 
FRONER, Y.A. Demandas históricas: a constituição da Ciência da Conservação e a formação do Conservador-Restaurador, Conservar Património 23, 2016, p.15-23. doi:10.14568/cp2015048,

http://revista.arp.org.pt/pdf/2015048.pdf (acesso em 201610-10).

ICCROM. Conservation Science. http://www.iccrom.org/wpcontent/uploads/YSIC I 60_S2 combined.pdf. (acesso em 2016-10-10)

JOKILEHTO, J., A History of Architectural Conservation, tese, England: University of York, 1986 http://www.iccrom.org/ifrcdn/pdf/ICCROM_05_HistoryofCon servation01_en.pdf (acesso em 2015-09-21)

LAURENTE, S., Les Arts appliqués en France. Genèse d'un enseignement, Paris : Éditions du CTHS, 1999.

LEVEAU, P. Le souvenir de la Grande Guerre dans les réseaux de conservation de l'Entre-Deux-Guerres. une préhistoire du bouclier bleu, In Situ 23, 2014, doi: 10.4000/insitu.10903.

LEVEAU, P. Épistémologiede la conservation-restauration, Provence: Université de Provence, 2012.

LEVEAU, P., Problèmes historiographiques de la conservationrestauration des biens culturale', CRBC 26, 2008, p. 3-18.

LEVEAU, P., L'enquête sur la formation des restaurateurs dans I'Entre-Deux-Guerres: transformation d'un métier et reconnaissance d'une profession (1929-2011), CeROArt, 9, 2014, http://ceroart.revues.org/3772. (acesso em 2015-0921)

MAURER, R.;Rinnebach, H.,La défense du patrimoine artistique et la formation des restaurateurs, Mouseion, 20 (4), 1932, p.142-146

MORIN, Edgar. A cabeça bem-feita: repensar a reforma, reformar o pensamento, RJ: Bertrand, 2003.

MORIN, Edgar. La mission de l'intellectuel', in L'intellectuel, l'intelligentsia et les manuels, ed. BELKIK, Paris :Anthropos, 1983, p.20-39

OIM, L'Activite de l'Office International des Musées, Mouseion, 10 (1), 1930, p.77-81.

OIM, L'Activite de l'Office International des Musées, Mouseion, 11 (2), 1930, p.184-210.

OIM, Suppléments, Mouseion, 13-14 (1-2), 1931, p. 192-203.

PLENDERLEITH, H.J. Preservation of Museum Objects in War-time. Nature 152, p.94-97, doi: 10.1038/152094a0.
PLENDERLEITH, H.J., A history of conservation, Studies in Conservation 43 (3), 1998, p. 129-143, doi: 10.1179/sic.1998.43.3.129.

PRICE, N. S., Preface, in Historical and Philosophical Issues in the Conservation of Cultural Heritage, ed. N. S. PRICE; M. K. TALLEY JR. E A. M. VACCARO, Los Angeles: GCI, 1996, x-xvi.

SCHAPIRO, Meyer. The New Viennese School, Art Bulletin 18, 1936, p.258-266.

SCHMIDT-DEGENER, F; SANCHES CANTON, F.J; MACLAGAN, E.; FOUNDOUKIDIS, E., Museographie: architecture et aménagement des musées d'art, Vol I e II, Paris: International Institute of Intellectual Co-operation-Office International des Musées, 1935

SEASE, C., A short history of archaeological conservation, in Archaeological Conservation and its Consequences, ed. A. ROY, P. SMITH, London: IIC, London, 1996), p. 157-161.

UNESCO Archives AtoM Catalogue. 'Conservation des oeuvres d'art. Protection des établissements et monuments d'art en temps de guerre', 4 volumes de documentos (19301936/1937-1938/janvier-juillet 1939/1939-janvier 1946).

VACCARO, A. M., The emergence of modern conservation theory, ed. N. S. PRICE; M. K. TALLEY JR. E A. M. VACCARO, Los Angeles: $\mathrm{GCl}, 1996$, p.202-211

WAETZOLDT, W.; GRAUL, R., La défense du patrimoine artistique et la formation des restaurateurs, Mouseion, 21-22 (1-2), 1933, p.269-270.

WHITMORE, P. The 2011 Robert L. Fellow Award: Presentation Remarks, (2011) http://www.heritagescience.ac.uk/ resources/publications/Conservation_science_report.pdf(ace ssoem 2015-09-21).

Contribuição ao

1‥ Simpósio Brasileiro de Caracterização e Conservação da Pedra 14 a 16 de dezembro de 2016, Congonhas - MG

\section{Nota:}

É de responsabilidade da comissão editorial do Simpósio a revisão gramatical, ortográfica, de citações e referências bibliográficas. As normas de submissão podem se diferenciar das desta revista. 